\title{
Highlights for Improvement of Scientific Writing for Publication in High Impact Journals
}

\author{
William W. Au*
}

University of Texas Medical Branch, USA; Shantou University Medical College, China; and University of Medicine and Pharmacy at Tirgu Mures, Romania

For research scientists around the world, a primary goal is to publish results from their projects in high impact international journals. Such an achievement can be highly rewarding because it is a formal way to release discoveries to the world and to be recognised for the discoveries, it allows findings to be shared and used by colleagues, and it can bring in personal benefits in awards and promotions. However, achieving the goal is not a simple task, and it can sometimes be frustrating. Therefore, this editorial was written to provide some highlights on how to improve chances for high impact publications and recognitions.

In writing this editorial, I draw on my experience as the Editor of the International Journal of Hygiene and Environmental Health which has an impact factor of 4.6 and which is ranked in the top $15 \%$ of international journals among toxicology, environmental health and public health. Also, I have published more than 200 scientific papers, book chapters and monographs in highimpact journals.

The topic of scientific writing and publication has been written in many books, and I have listed two books here as references (Keshavan, 2014; Olson, 2015). Certainly, these and other books recommend procedures and criteria that have been well-tested and accepted for their usefulness. For example, it is necessary to provide strong justification for research projects, to present results concisely and attractively, to submit conclusions without exaggeration, etc. These golden rules should be followed, but other criteria which are helpful can be considered. These criteria are presented particularly for those whose native language is not English.

It is evident that writing a paper for publication is dictated by the experimental design of the project and by the availability of the collected data. Nevertheless, authors should write the paper focusing on five criteria which are shown below. The criteria are needed because reviewers and readers have no idea about what the authors have done and what value the paper pro- vides. Therefore, authors must work hard to identify information that is relevant to the criteria. Consequently, reviewers and readers will be convinced that the work is significant.

1. The hypothesis is original;

2. The aims are innovative;

3. The techniques are up to date and useful;

4. The results are not ambiguous and can be easily understood;

5. The conclusion is meaningful and useful.

Another major consideration is the title of the paper. Since the title is the first information to be observed by reviewers and readers, it sets the first impression on the paper, whether enthusiastic or unenthusiastic. Therefore, the title needs to be highly concise, informative and attractive. As an example, consider a title "Development of an assay to detect breast cancer in the early stages". Well, the same title can be rewritten as "An innovative assay for early breast cancer detection". Although both titles provide the same information, the second title is more concise and more attractive.

After the title, the abstract is the second most important component of a paper because it is a concise summary of the entire paper. Well-written abstracts will certainly influence favourable decisions by reviewers.

Nowadays, scientists conduct searches on the internet for publications of their interest. During and after the search, scientists typically read abstracts of the selected publications and decide whether a publication is worthwhile for further consideration or not. In reality, when abstracts are well-written, many scientists may not read the rest of publications and will rely on information that is provided in abstracts. Therefore, one can consider that abstracts can determine the fates of publications because these publications may be cited solely based on information that is presented in excellent abstracts. Therefore, abstracts must contain data and appropriate information, and be highly convincing. 
The first two sentences of an abstract are important because they quickly attract the attention of reviewers and readers. Typically, the first sentence can be written to show the importance of the scientific problem and the second sentence to show innovative findings from the investigation. Here are three excellent examples for consideration:

1. "Infection with H6N9 influenza virus is an emerging infectious disease, and it is highly deadly. Our investigation of 50 infected patients and 50 resistant controls show that ...... was a key cell membrane receptor which controlled infectivity of the virus".

2. "Exposure to chemicals from cigarette smoking is the main cause of lung cancer, but their toxicity to developing embryo has not been well-characterized. We have identified a molecular pathway for cell differentiation which was specifically interrupted by such chemicals in cells from amniotic fluid of mothers who had been cigarette smokers".

3. "Electrocardiogram which is the main screening test for left ventricular hypertrophy has low sensitivity. Our investigation on sixty patients shows that a specific modification of electrocardiogram improved the sensitivity by $30 \%$ ".

In these three examples, the remainder of each abstract was used to provide experimental data and to explain the findings. In these cases, readers were prompted to read the abstracts in full, to review and consequently to cite each paper.

For non-native English-speaking scientists, it is necessary that their papers are reviewed by English-speaking specialists before submitting the papers for consideration for publication because poor English writing is frequently a cause for rejection of papers. For example, the use of the word "the" indicates certain specificity. The title, as shown below, uses the word "the" incorrectly because it forced reviewers/readers to think about what specific "role" and "pathogenesis" were involved. Since the title introduced the topic for the first time, it became a futile effort for reviewers and readers to try to identify what specificities were conveyed by the authors. Consequently, they became discouraged from reading the abstract and the paper.

"The role of metabolic biomarkers in the pathogenesis of colon cancer". Therefore, the title should be rewritten as "Involvement of metabolic biomarkers in pathogenesis of colon cancer". If the collected data are new and exciting, one can rewrite the title as "Unique involvement of metabolic biomarkers in pathogenesis of colon cancer". This title would generate more attention than the other two.
A frequent and major problem for non-native English writers is the misuse of past, present and future tenses. These have specific meanings. Past tenses are used to indicate what has been done, either in published papers or the current paper. For example, "we found that high body mass indices were significantly associated with stroke $(\mathrm{p}<0.05)$ ". Present tenses are used to indicate well-established knowledge, e.g. "the earth is round"; "routine and well-managed exercises are beneficial to health". Future tenses are used to indicate what will be done in the future, e.g. "our observed discrepancies will be investigated in our ongoing experiments".

Serious confusions occur when the tenses are misused. For example:

"False positive and false negative influenza testing may be reported, leading to erroneous patient's management. Therefore, we perform antigenic ELISA assays to improve sensitivity which showed decisive impact on therapy and follow-up."

The paragraph above contains serious grammatical errors but also problems in expressing meanings, e.g. exaggeration. One way to improve the writing can be:

"False positive and false negative influenza testing have been reported and have caused erroneous management of patients. Therefore, we have performed antigenic ELISA assays to improve sensitivity of the test. Our results show that the test can be used to improve therapeutic outcomes".

It is clear that every component of a paper needs to be written well. However, authors should also pay particular attention to meeting certain criteria and to emphasising priority areas, e.g. abstracts and grammar. The combination of these efforts will improve the chances of publishing papers in high impact journals.

\section{NOTE}

Statements in quotations within this editorial are from the author and are not taken from any publications.

\section{CONFLICT OF INTEREST}

None to declare.

\section{REFERENCES}

- Keshavan N. Medical and Scientific Writing. Kindle Edition, Kindle Unlimited, UK, 2014.

- Olson, L. How to Get Your Writings Published in Scholarly Journals. Academic Press, NY, USA, 2015. 